\title{
Noninvasive follicular thyroid neoplasm with papillary-like nuclear features and the risk of malignancy in thyroid cytology: Data from Singapore
}

Bryan Lee Wei Wen ${ }^{1,7}{ }_{M B B S}$, Manish Mahadeorao Bundele ${ }^{4}{ }_{\text {FRCPath }}$, Rong $\underline{\text { Tan }}^{2,7}{ }_{M B B S}$, Ernest Fu Wei Zhong ${ }^{3}{ }_{\text {FAMS (ORL), }}$ Agnes Chew Siqi ${ }^{3} B A$, Junice Wong Shi Hui ${ }^{5}{ }_{F R C S E d}$, Caroline Siew Ching Hsia ${ }^{5}{ }_{F R C S E d}$, Brenda Lim Su Ping ${ }^{6}$ FRCP, Rinkoo Dalan ${ }_{F R C P}$, Ming Yann $\underline{\operatorname{Lim}}^{3}{ }_{F A M S}\left(\right.$ ORL), Yijin Jereme $\underline{\operatorname{Gan}}^{3}{ }_{F A M S}(O R L)$, Hao $\underline{\operatorname{Li}}^{3}{ }^{3}{ }^{3}$

\begin{abstract}
Introduction: The impact of noninvasive follicular thyroid neoplasm with papillary-like nuclear features (NIFTP) on the risk of malignancy (ROM) in fine-needle aspiration cytology (FNAC) per The Bethesda System for Reporting Thyroid Cytopathology has not been well reported in Singapore.

Methods: We retrospectively identified 821 thyroid nodules with preoperative FNAC from 788 patients out of 1,279 consecutive thyroidectomies performed between January 2010 and August 2016 in a tertiary general hospital in Singapore. Possible cases of NIFTP were reviewed for reclassification and the impact of NIFTP on ROM was analysed.

Results: The incidence of NIFTP was $1.2 \%$ (10 out of 821 ). If NIFTP is considered benign, ROM in Bethesda I through VI were $8.6 \%, 3.5 \%, 26.3 \%, 20.0 \%, 87.7 \%, 97.0 \%$ versus $8.6 \%, 4.2 \%, 28.1 \%$, $26.7 \%, 89.2 \%$ and $100 \%$ if NIFTP is considered malignant. Eight patients with NIFTP had follow-up of 15 to 110 months. One had possible rib metastasis as evidenced by $\mathrm{I}^{131}$ uptake but remained free of structural or biochemical disease during a follow-up period of 110 months. None had lymph node metastasis at presentation, nor locoregional or distant recurrence.

Conclusion: Classifying NIFTP as benign decreased ROM in Bethesda II through VI, but the benignity of NIFTP requires more prospective studies to ascertain. The impact of NIFTP on ROM in our institution also appears to be lower than that reported in the Western studies.
\end{abstract}

Ann Acad Med Singap 2021;50:903-10

Keywords: Bethesda, cytology, NIFTP, risk of malignancy, TBSRTC, thyroid nodule

\section{INTRODUCTION}

As only $5-10 \%$ of thyroid nodules harbour malignancy, fine-needle aspiration (FNA) is important in triaging nodules requiring surgical excision from nodules that may be observed. ${ }^{1,2}$ This approach allows risk stratification that can reduce the rate of benign lesion removal by diagnostic lobectomies ${ }^{3}$ and its associated postoperative morbidity. ${ }^{4}$ However, thyroid cytology reports should convey a reproducible risk of malignancy (ROM) in order to facilitate surgical decision making. The Bethesda System for Reporting Thyroid Cytopathology
(TBSRTC), published in $2009,{ }^{5}$ is a 6-tier system with standardised nomenclature. Using TBSRTC, cytopathologists can report FNAs to the referring physician in terms that are succinct, unambiguous and clinically useful. ${ }^{5}$ In 2017, the second edition of TBSRTC $^{6}$ was published, in which ROM was revised especially for the indeterminate categories and according to the benignity of noninvasive follicular thyroid neoplasm with papillary-like nuclear features (NIFTP). NIFTP is a subgroup of noninvasive/ encapsulated follicular variant papillary thyroid carcinoma

\footnotetext{
${ }^{1}$ Lee Kong Chian School of Medicine, Nanyang Technological University, Singapore

${ }^{2}$ Yong Loo Ling School of Medicine, National University of Singapore, Singapore

${ }^{3}$ Department of Otorhinolaryngology, Tan Tock Seng Hospital, Singapore

${ }^{4}$ Department of Pathology, Tan Tock Seng Hospital, Singapore

${ }^{5}$ Department of General Surgery, Tan Tock Seng Hospital, Singapore

${ }^{6}$ Department of Endocrinology, Tan Tock Seng Hospital, Singapore

${ }^{7}$ Ministry of Health Holdings, Singapore

Correspondence: Dr Hao Li, Department of Otorhinolaryngology, Tan Tock Seng Hospital, 11 Jalan Tan Tock Seng, Singapore 308433.

Email:Li_Hao@ttsh.com.sg
} 


\section{CLINICAL IMPACT}

\section{What is New}

- This study from Tan Tock Seng Hospital in Singapore evaluates the impact of noninvasive follicular thyroid neoplasm with papillary-like nuclear features (NIFTP) on the risk of malignancy in thyroid fine-needle aspiration cytology categorised by the Bethesda system.

\section{Clinical Implications}

- Understanding the risk of malignancy in thyroid cytology and the impact of NIFTP in the Singapore context can help clinicians manage patients with thyroid nodules more appropriately.

(PTC) that can be treated as indolent, perhaps benign, tumours. It is diagnosed on histopathology using strict criteria first proposed by Nikiforov et al. ${ }^{7}$ NIFTPs are known to have a very low recurrence rate (near zero), ${ }^{8}$ therefore its diagnosis may reduce the patient's psychological burden and decrease over-treatment. However, little is known in terms of the ROM per TBSRTC in a Singapore patient population and how the introduction of NIFTP may affect the ROM. Such information should be sought in order to help clinicians in Singapore counsel their patients more precisely on the implications of their FNA results. Worldwide, the impact of NIFTP on ROM depends on the incidence of NIFTP in each population. Several studies from different countries and institutions including a recent meta-analysis showed a wide variation in the prevalence of NIFTP among lesions diagnosed as PTC. ${ }^{9-12}$ In this study, we aim to determine the ROM in various Bethesda categories in the setting of a tertiary general hospital in Singapore and how NIFTP would affect ROM in a multiethnic Southeast Asian population.

\section{METHODS}

After approval from the Domain Specific Review Board of National Healthcare Group, Singapore (reference number 2017/01150), we retrieved electronic medical records of patients who underwent hemithyroidectomies, total thyroidectomies or completion thyroidectomies in the Department of Otorhinolaryngology or General Surgery between January 2010 and August 2016 in Tan Tock Seng Hospital, Singapore. Out of 1,279 patients, 491 were excluded as they did not have preoperative thyroid FNA or the FNA report was not available, or the site of FNA could not be determined. This left 788 patients with 821 FNAs on different thyroid nodules, in whom we could match the cytology to histology. Matching of preoperative FNA to postoperative histology was performed by the senior author (HL) and co-author (RT). The senior author verified all matching performed by the co-author and resolved all queries. Special attention was paid to papillary thyroid microcarcinomas (PTMCs). The senior author reviewed all PTMCs to determine if they were subjected to FNA. PTMC was matched to the cytology if FNA was ultrasoundguided and the site of FNA matched the site of PTMC on the histopathology of the thyroidectomy specimen. Otherwise, the PTMC was considered incidental. As FNA was performed under ultrasound guidance (USG) in $58.3 \%$ of nodules (479 out of 821 ), close attention was also paid to the matching of FNAs that were not performed under USG. A third author (BL) reviewed the medical records to verify the matching. In most of these nodules (326 out of 342), the clinicians stated that they performed the FNA on the palpable or dominant nodule and indicated the location of the FNA. In the other 16 patients, no nodule was clearly dominant, but the histology of the thyroid gland showed benign multinodular goitre or lymphocytic thyroiditis uniformly throughout the thyroidectomy specimen. Therefore, their cytology could be matched to these diagnoses. In addition, we collected each patient's age at thyroidectomy, sex and ethnicity. For patients with NIFTP, the size of NIFTP, postoperative treatment, results of structural and biochemical follow-up were also collected to determine the occurrence of locoregional recurrence or distant metastasis.

\section{Cytological preparation}

FNA was performed using a 23- or 25-gauge needle attached to a disposable plastic syringe. Samples obtained were expelled onto 4 to 8 glass slides and smeared. Half of these smears were placed in $95 \%$ alcohol for Papanicolaou staining and the other half left to air dry before Giemsa or Hemacolor staining. Cell block preparations were made when adequate material was available. Cytotechnologists were routinely on site to assess the adequacy of FNA samples.

\section{Classification of cytology and histology}

FNA specimens were interpreted by pathologists from our institution using TBSRTC ${ }^{5,6}$ and categorised into 1 of 6 categories: (I) nondiagnostic or unsatisfactory, (II) benign, (III) atypia of undetermined significance or follicular lesion of undetermined significance (AUS or FLUS), (IV) follicular neoplasm, (V) suspicious for malignancy, and (VI) malignant. For the purpose of this study, all cytology reports were reviewed again by a head 
and neck pathologist (second author MMB) to confirm the Bethesda category. This pathologist also reviewed the histological reports of all thyroid nodules and standardised the diagnostic nomenclature according to the 2017 World Health Organization classification of endocrine tumours. ${ }^{13}$

\section{Reclassification of NIFTP}

To identify NIFTP, histological slides of all follicular or Hürthle cell adenomas, adenomatoid nodule with atypia, follicular neoplasm of uncertain malignant potential, and follicular variant papillary thyroid carcinomas (FVPTCs) were reviewed by MMB and reclassified as NIFTP if they fulfill the criteria described by Nikiforov et al., ${ }^{7}$ as follows:

\section{Inclusion criteria}

The tumour is:

- Encapsulated or well demarcated

- Follicular patterned

- Demonstrates at least focal nuclear features of papillary thyroid carcinoma

\section{Exclusion criteria}

- Infiltration/tumoural capsular invasion

- Solid/trabecular or insular growth greater than $30 \%$

- True papillary growth (with fibrovascular cores) (even 1 well-formed papillary structure)

- Psammoma bodies

- Tall cell, hobnail cell, columnar cell, or cribriform morular morphology

- Necrosis

- Mitoses greater than 3 per 10 consecutive high-power fields (HPF) (in solid/microfollicular areas)

Entire nodule and complete tumour-capsule interface of encapsulated nodules or nodules with atypia indeterminate of papillary thyroid carcinoma were routinely evaluated in our Department of Pathology prior to 2016. Therefore, they are sufficient for the definitive diagnosis of NIFTP in this study.

\section{Follow-up}

Patients diagnosed with thyroid cancer postoperatively were routinely followed up with thyroglobulin and antithyroglobulin antibodies, free T4, thyroid stimulating hormone (TSH), structural imaging of the neck, and prescribed levothyroxine for TSH suppression if the cancer was papillary or follicular carcinoma. Response to treatment was classified according to the guidelines of American Thyroid Association ${ }^{19}$ and patients with biochemical indeterminate or incomplete response without structural neck disease would be referred for consideration of radioactive iodine.

\section{Statistical evaluation}

Statistical analysis was performed using STATA SE version 14.2 (StataCorp, College Station, US). Categorical data were tabulated as frequencies and percentages. Continuous data were summarised as mean, median and range. Shapiro-Wilk test was performed to assess normality. Fisher's exact test was performed to compare categorical variables. All $P$ values were 2 -sided and $P<0.05$ was considered significant.

\section{RESULTS}

\section{Demography and histology}

Age at thyroidectomy ranged from 14 to 88 years with a median of 52 years. There was female predominance for malignant pathology/incidental PTMC (78.4\%) and benign pathology/NIFTP (79.8\%). The distribution of ethnicity in patients with malignant pathology/ incidental PTMC was $71.2 \%$ Chinese, $10.2 \%$ Malay, $7.2 \%$ Indian and $11.4 \%$ others, while that for benign pathology/NIFTP was $80.2 \%$ Chinese, $7.5 \%$ Malay, $5.5 \%$ Indian and $6.8 \%$ others. Table 1 shows the histological diagnoses of the thyroid nodules subjected to FNA: $22.7 \%$ were malignant, $76.1 \%$ were benign and $1.2 \%$ were NIFTPs. The most common malignancy subjected to FNA was conventional-type papillary thyroid carcinoma $(122 / 186,65.6 \%)$ followed by follicular thyroid carcinoma $(36 / 186,19.4 \%)$. The most common benign lesion subjected to FNA was nodular or multinodular goitre $(563 / 625,90.1 \%)$ followed by follicular or Hürthle cell adenoma (34/625, 5.4\%).

\section{Histopathology of NIFTP}

Upon review of the 16 FVPTCs, 3 were reclassified as NIFTP, 3 were encapsulated noninvasive FVPTCs and 10 were encapsulated invasive FVPTCs. The 3 encapsulated, noninvasive FVPTCs were not NIFTP as focal true papillae formation was present, and one showed $>30 \%$ solid/trabecular pattern. There were 38 follicular adenomas, of which 5 showed atypia, raising concern of papillary thyroid carcinoma. Upon review, these were classified as NIFTP. One adenomatoid nodule with atypia and 1 follicular neoplasm with uncertain malignant potential was also reclassified as NIFTP (Fig. 1).

\section{Clinical features of NIFTP}

Table 2 shows the clinical features of the 10 NIFTP diagnosed retrospectively. Cytologically, 1 was 
Table 1. Histological diagnoses of all thyroid nodules that underwent fine-needle aspiration (FNA)

\begin{tabular}{|c|c|}
\hline Histology & $\begin{array}{c}\text { Number of nodules }(\%) \\
\text { Total }=821\end{array}$ \\
\hline Malignant & $186(22.7)$ \\
\hline Papillary thyroid carcinoma (PTC) & $135(16.4)$ \\
\hline Conventional ${ }^{a}$ & $106(12.9)$ \\
\hline Follicular variant PTC (FVPTC) & $13(1.6)$ \\
\hline - Encapsulated invasive & $10(1.2)$ \\
\hline - Encapsulated non-invasive & $3(0.4)$ \\
\hline Microcarcinoma $^{\mathrm{b}}(\mathrm{PTMC})$ biopsied by FNA & $16(1.9)$ \\
\hline Follicular carcinoma & $36(4.4)$ \\
\hline Minimally invasive & $21(2.6)$ \\
\hline Encapsulated angioinvasive & $8(1.0)$ \\
\hline Widely invasive & $7(0.9)$ \\
\hline Oncocytic (Hürthle cell) carcinoma & $7(0.9)$ \\
\hline Encapsulated angioinvasive & $6(0.8)$ \\
\hline Widely invasive & $1(0.1)$ \\
\hline Medullary carcinoma & $5(0.6)$ \\
\hline Poorly differentiated carcinoma & $1(0.1)$ \\
\hline Anaplastic carcinoma & $1(0.1)$ \\
\hline Metastatic adenocarcinoma & $1(0.1)$ \\
\hline Noninvasive follicular thyroid neoplasm with papillary-like nuclear features & $10(1.2)$ \\
\hline Benign & $625(76.1)$ \\
\hline Multinodular goitre/Nodular thyroid hyperplasia & $515(62.8)$ \\
\hline Follicular/Hürthle cell adenoma & $33(4.0)$ \\
\hline Lymphocytic or Hashimoto thyroiditis & $21(2.6)$ \\
\hline Benign cyst & $4(0.5)$ \\
\hline Granulomatous thyroiditis & $1(0.1)$ \\
\hline Graves' disease & $1(0.1)$ \\
\hline Incidental ${ }^{\mathrm{C}}$ papillary thyroid microcarcinoma & $50(6.1)$ \\
\hline \multicolumn{2}{|l|}{ Actual diagnoses of the nodule biopsied by FNA } \\
\hline - Multinodular goitre/Nodular thyroid hyperplasia & $48(5.8)$ \\
\hline - Follicular/Hürthle cell adenoma & $1(0.1)$ \\
\hline - Lymphocytic or Hashimoto thyroiditis & $1(0.1)$ \\
\hline
\end{tabular}

FNA: fine-needle aspiration; PTMC: papillary thyroid microcarcinoma

${ }^{a}$ Larger than $1 \mathrm{~cm}$ in the longest dimension

${ }^{\mathrm{b}} \mathrm{Up}$ to $1 \mathrm{~cm}$ in the longest dimension

${ }^{\mathrm{c}}$ Not the nodule biopsied by fine-needle aspiration

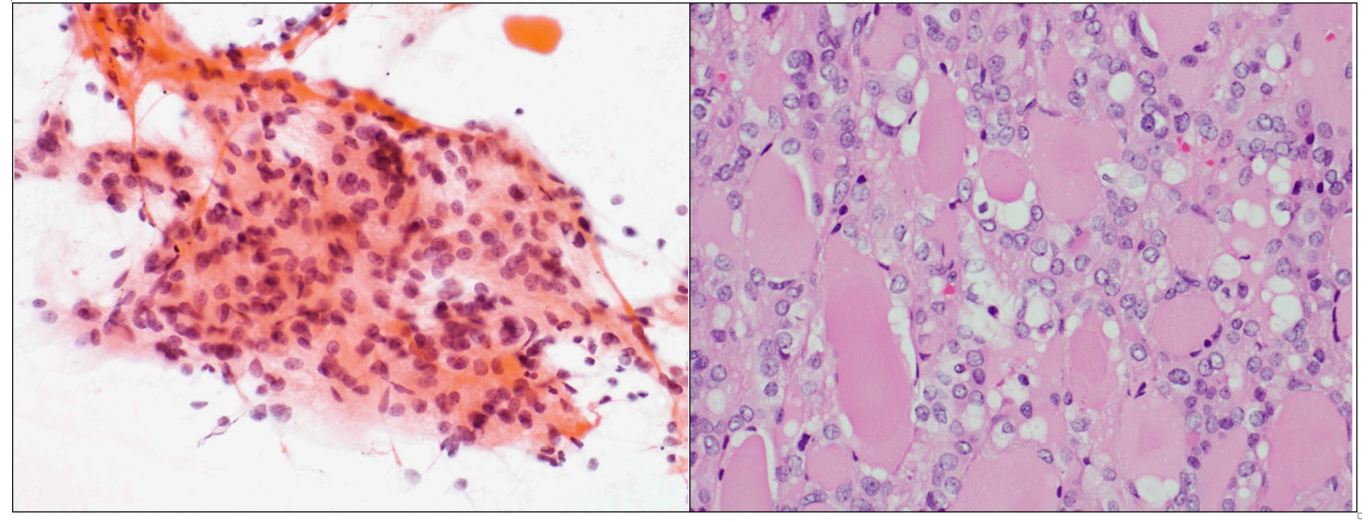

A

B

Fig. 1. (A) Papanicolaou (PAP) stain of the cytology and (B) corresponding haematoxylin and eosin stain of the histology from one of the patients diagnosed with NIFTP. Clusters of follicular cells with nuclear atypia featuring enlarged nuclei with pale chromatin and nuclear groves can be seen with PAP stain, while histology of the nodule showed follicular patterned cells with nuclear enlargement, clearing, membrane irregularities and grooves. 
diagnosed as suspicious of PTC (Bethesda V) and 1 as PTC (Bethesda VI). Two were benign (Bethesda II) with no nuclear atypia of PTC seen. Out of the remaining 6 indeterminate cases (1 Bethesda IV and 5 Bethesda III), nuclear atypia of PTC was present in 5 cases. However, the quality and quantity of nuclear atypia was variable and features such as nuclear pseudoinclusions, papillae or psammoma bodies were absent. Histologically, the original diagnoses of these NIFTP were 5 follicular adenomas with atypia, 3 follicular variant PTCs (FVPTCs), 1 adenomatoid nodule with atypia and 1 follicular neoplasm of uncertain malignant potential. The median duration of follow-up was 61 months. Two patients were lost to follow-up. In 1 of the 3 patients originally diagnosed as FVPTC, postoperative $\mathrm{I}^{131}$ whole-body-scan showed uptake in the rib without structural lesion on SPECT-CT. This was suggestive of bone metastasis. However, he remained free of structural or biochemical disease over a follow-up of 110 months after $\mathrm{I}^{131}$. The other 2 patients had either biochemically indeterminate or excellent response without structural recurrence. Therefore, no patient with NIFTP developed locoregional recurrence or distant metastasis during follow-up. Moreover, the sonographic features of 7 out of 10 NIFTP could be ascertained. All were solid to predominantly solid but the echogenicity varies from hyperechoic to markedly hypoechoic, with 3 showing heterogenous echogenicity. Six had colour Doppler sonography and all showed internal vascularity. None had features associated with high likelihood of malignancy, namely, taller-than-wider orientation, microcalcification, irregular margin, extrathyroidal extension or lymphadenopathy.

\section{Risk of malignancy in FNA cytology and the impact of NIFTP}

Table 3 describes histologically proven ROM in each Bethesda category compared to the reference ranges published by TBSRTC in 2017. ${ }^{6}$ Frequency of cytological categories in decreasing order are: benign $(35.0 \%)$, AUS or FLUS $(32.9 \%)$, non-diagnostic or unsatisfactory $(18.4 \%)$, suspicious for malignancy (7.9\%), malignant $(4.0 \%)$, and follicular neoplasm or suspicious for follicular neoplasm (1.8\%). ROM per Bethesda category increases sequentially similar to the reference ranges in TBSRTC. When NIFTP is considered benign, ROM in our study was higher than the reference ranges in Category II (3.5\%), III (26.3\%), V (87.7\%), VI (97\%), and within the reference ranges in Category I $(8.6 \%)$ and IV $(20.0 \%)$. When NIFTP is considered malignant, ROM increases by $0 \%, 0.7 \%$, $2.2 \%, 7 \%, 1.5 \%$ and $3 \%$ in each Bethesda category, respectively, and falls mostly within the reference range, except in category II (4.2\%), V (89.2\%) and VI $(100 \%)$, in which our ROM was slightly higher.

\section{DISCUSSION}

We present here data from Singapore on the risk of malignancy (ROM) in each Bethesda category of thyroid cytology and how NIFTP impacts the ROM. Considering NIFTP benign, our ROM was noticeably higher than the reference range of TBSRTC in Bethesda Category III. Several reasons can explain this. First, in this surgical series, there could be selection bias as surgeons consider not only cytology but also sonographic features, size, symptoms or signs of malignancy when recommending thyroidectomy. Second, the lack of ultrasound guidance in some of the FNAs can result in geographic miss in the sampling of the malignant portion of the nodule, leading to higher rates of indeterminate cytology.

With regards to the frequency of diagnosis per Bethesda category, we found a higher proportion of category III, $32.9 \%$, compared to $10.5 \%$ in a multiinstitutional Asian study conducted by Bychkov et al. ${ }^{11}$ Although TBSRTC recommends limiting frequency of category III (AUS/FLUS) to less than 10\%, ${ }^{4}$ reproducibility in AUS/FLUS is at best fair, ${ }^{14}$ and studies with low AUS/FLUS rates can have a relatively higher number of false negatives or positives depending on which categories these lesions with borderline cytomorphology get classified. ${ }^{14}$ Our study did not have false positives for category VI, our ROM in category $\mathrm{V}$ was higher than the reference range of TBSRTC and the frequency of category IV was low $(1.8 \%)$. These suggest a low threshold of classifying cytologically indeterminate or suspicious nodules into AUS/FLUS in our institution.

Since AUS/FLUS is common in our practice, refining ROM in AUS/FLUS is important. Gan et al. ${ }^{15}$ and Lim et al. ${ }^{16}$ subclassified AUS/FLUS into architectural or nuclear atypia. For lesions with architectural atypia, repeating FNA or clinical follow-up may be sufficient whereas surgery may be favoured in lesions with nuclear atypia given the higher ROM. Molecular testing may also refine ROM in indeterminate categories. ${ }^{17,18} \mathrm{However}$, the cost of molecular testing is preventing its routine use in all indeterminate cases. How to select a Bethesda III or IV nodule for molecular testing in a cost-effective way deserves more research.

The introduction of NIFTP in 2016 has implications on the management of thyroid nodules and influenced the ROM in each Bethesda category, especially in categories III and IV. ${ }^{19,20}$ However, the prevalence of NIFTP and the impact of NIFTP reclassification on the 


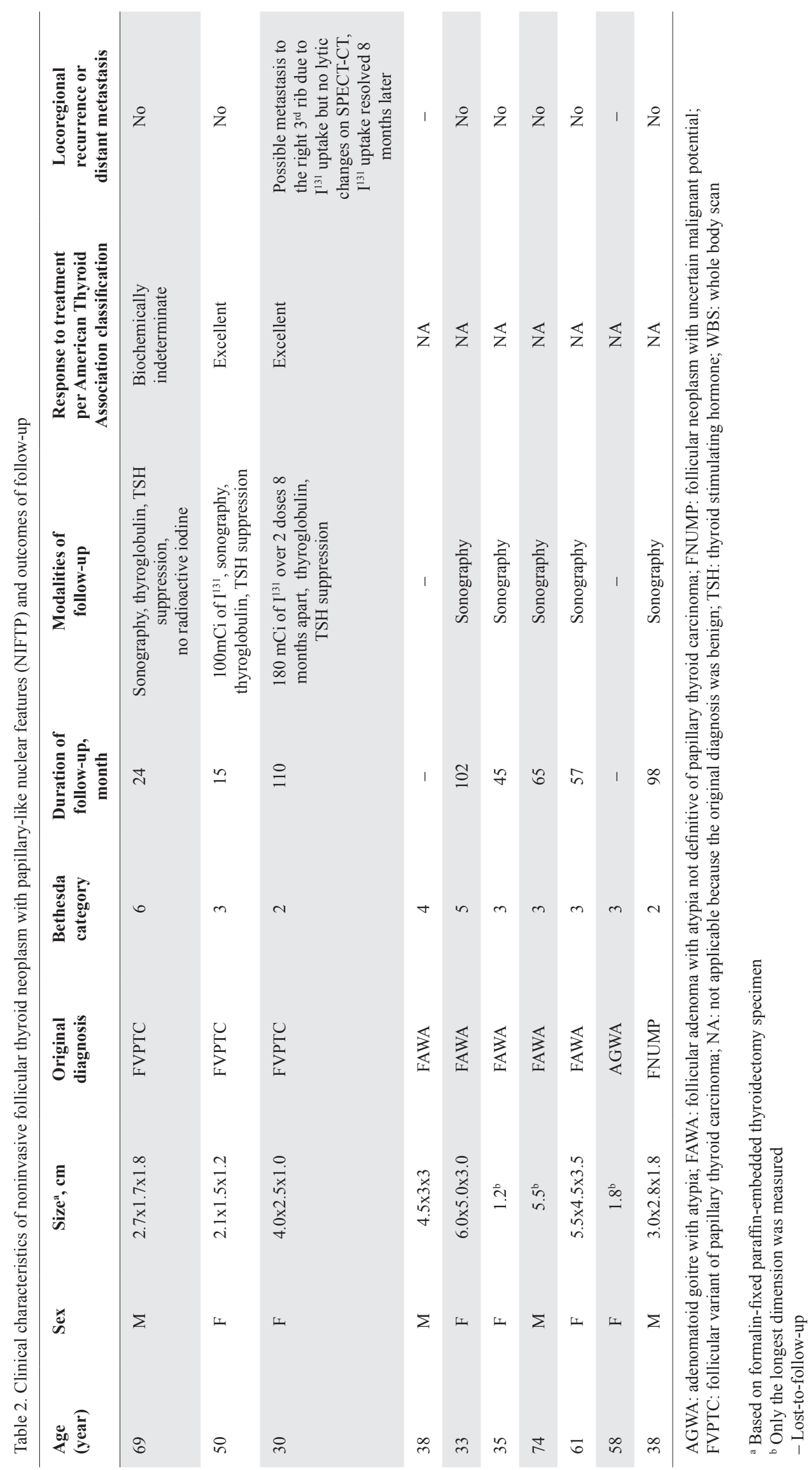


Table 3. Comparing the risk of malignancy (ROM) of thyroid cytology in our institution with the Bethesda reference range, stratified by benignity of NIFTP

\begin{tabular}{|c|c|c|c|c|c|}
\hline Bethesda category & $\begin{array}{c}\text { Number of } \\
\text { nodules }(\%) \\
\text { Total }=821\end{array}$ & $\begin{array}{l}\text { ROM if NIFTP is } \\
\text { malignant, } \\
\% \text { (no. of cancer) }\end{array}$ & $\begin{array}{l}\text { ROM if NIFTP is } \\
\text { benign, } \\
\% \text { (no. of cancer) }\end{array}$ & $\begin{array}{l}\text { ROM quoted by the } \\
2017 \text { TBSRTC, if } \\
\text { NIFTP is malignant } \\
(\%)\end{array}$ & $\begin{array}{c}\text { ROM quoted by the } 2017 \\
\text { TBSRTC, if NIFTP is } \\
\text { benign }^{\text {a }(\%)}\end{array}$ \\
\hline $\begin{array}{l}\text { I. Non-diagnostic or } \\
\text { unsatisfactory }\end{array}$ & $151(18.4)$ & $8.6(13)$ & $8.6(13)$ & $5-10$ & $5-10$ \\
\hline II. Benign & $287(35.0)$ & $4.2(12)$ & $3.5(10)$ & $0-3$ & $0-3$ \\
\hline III. AUS or FLUS & $270(32.9)$ & $28.1(76)$ & $26.3(71)$ & $10-30$ & $6-18$ \\
\hline $\begin{array}{l}\text { IV. Follicular neoplasm or } \\
\text { suspicious for follicular } \\
\text { neoplasm }\end{array}$ & $15(1.8)$ & $26.7(4)$ & 20.0 (3) & $25-40$ & $10-40$ \\
\hline $\begin{array}{l}\text { V. Suspicious for } \\
\text { malignancy }\end{array}$ & $65(7.9)$ & $89.2(58)$ & $87.7(57)$ & $50-75$ & $45-60$ \\
\hline VI. Malignant & $33(4.0)$ & $100(33)$ & $97.0(32)$ & $97-99$ & $94-96$ \\
\hline
\end{tabular}

AUS: atypia of undetermined significance; FLUS: follicular neoplasm of undetermined significance; NIFTP: noninvasive follicular thyroid neoplasm with papillary-like nuclear features; TBSRTC: The Bethesda System for Reporting Thyroid Cytopathology

${ }^{a}$ Cibas ES, Ali SZ. The 2017 Bethesda System for Reporting Thyroid Cytopathology. Thyroid 2017;27:1341-6.

Bethesda categories may differ in various populations. A recent meta-analysis by Rana et al. ${ }^{12}$ found the overall incidence of NIFTP to be $6.0 \%$ among PTCs or thyroid malignancies, with a significantly lower overall incidence in Asia (2.1\%) compared to North America or Europe (9.3\% and 9.6\%, respectively), and a multi-institutional Asian study by Bychkov et al. found NIFTP in only $2.9 \%$ of all excised nodules. ${ }^{11}$ To our best knowledge, studies from Singapore have yet to address the impact of NIFTP classification on the rates of malignancy in thyroid cytology. Our incidence of NIFTP from a tertiary hospital in Singapore was $5.1 \%$ among PTCs (10 NIFTP out of 195 PTCs and NIFTP). This was slightly higher than that reported by Bychkov et al. or Rana et al., but consistent with the wide variation in the incidence of NIFTP between Asian countries from $0.4 \%$ in $\mathrm{China}^{21}$ to $14.4 \%$ in India. ${ }^{22}$ Reasons for the discrepancy between Asian and Western studies may be multifactorial, including variation in the incidence of FVPTCs between continents, ${ }^{23}$ racial and ethnic predispositions to PTC, ${ }^{24}$ and varying threshold of diagnosing nuclear features of PTC.

In terms of the cytology of NIFTPs, we found that $50 \%$ were Bethesda III, 20\% Bethesda II, and 10\% each for Bethesda IV, V and VI. Our proportion of Bethesda IV and V are relatively low compared to a meta-analysis by Ruanpeng et al. ${ }^{20}$ and a multiinstitutional Asian study by Bychkov et al. ${ }^{11}$ In these studies, NIFTP appears to be most commonly Bethesda
III (34.2\%) or IV (22.7-32.2\%), followed by V (22.4\%). This difference is consistent with the relatively low frequency of Bethesda IV in our institution. Moreover, the proportion of Bethesda III also appears relatively high in our study, perhaps because we also reviewed cases of follicular adenoma and adenomatous nodule with atypia in addition to encapsulated follicular variant of PTC. As most of the studies on NIFTP were conducted in the US and Europe, our data contribute to a growing body of literature from Asia on the different impact of NIFTP on thyroid cancers in our region.

Finally, even though TBSRTC permits the classification of NIFTP as benign, the benignity of NIFTP is still debated. A recent Canadian series found that up to $6 \%$ of NIFTP patients had nodal or distant metastases, ${ }^{25}$ suggesting that NIFTP behaved as a low-risk thyroid cancer. One of our patients had possible iodine avid bone metastasis, although he was alive with an excellent response to therapy nearly 10 years since treatment. Nonetheless, we had few NIFTP patients and not all had long-term follow-up. Further studies worldwide are required to support the benignity of NIFTP.

Our study has several limitations. First, the estimation of ROM was based only on patients who underwent thyroidectomies. Inclusion of patients whose thyroid nodules are observed is necessary to estimate more accurately the ROM of Bethesda I to III. Second, this is a single institutional experience with a small number of NIFTP, therefore may not reflect the true impact of NIFTP on thyroid cytology in Singapore. Third, the 
retrospective matching of cytology to histology could lead to errors, but this error is likely random and the verification of matching mitigated it. The chance of error should also be balanced among the different cytological categories. Nonetheless, a prospective study may be done to further validate the ROM found in this study.

\section{CONCLUSION}

Our study describes the demography of patient with thyroid nodules who underwent FNA and thyroidectomy, the histology of their nodules, and the ROM per 2017 TBSRTC from a tertiary general hospital in Singapore. While TBSRTC is one of the most validated FNA classifications today, variation in ROM exists worldwide. Compared to Western studies, we found a higher frequency and ROM in the Bethesda III category but a lower impact of NIFTP on ROM. Understanding the risk of malignancy of thyroid cytology in the Singapore context can help clinicians more appropriately manage patients with thyroid nodules.

\section{Acknowledgement}

The authors would like to thank Sister Sow Fong Lee, the chief nurse of the Operating Theatre at Tan Tock Seng Hospital, for facilitating the data collection.

\section{REFERENCES}

1. Sakorafas GH. Thyroid nodules; interpretation and importance of fine-needle aspiration (FNA) for the clinician - practical considerations. Surg Oncol 2010;19:e130-9.

2. Tan WJ, Sanghvi K, Liau KH, et al. An audit study of the sensitivity and specificity of ultrasound, fine needle aspiration cytology and frozen section in the evaluation of thyroid malignancies in a tertiary institution. Ann Acad Med Singap 2010;39:359-62.

3. Yassa L, Cibas ES, Benson CB, et al. Long-term assessment of a multidisciplinary approach to thyroid nodule diagnostic evaluation. Cancer 2007;111:508-16.

4. Lee JC, Siow JK. Thyroid surgery--the Tan Tock Seng Hospital otolaryngology experience. Ann Acad Med Singap 2002;31:158-64.

5. Cibas ES, Ali SZ. The Bethesda System for Reporting Thyroid Cytopathology. Thyroid 2009;19:1159-65.

6. Cibas ES, Ali SZ. The 2017 Bethesda System for Reporting Thyroid Cytopathology. Thyroid 2017;27:1341-6.

7. Nikiforov YE, Seethala RR, Tallini G, et al. Nomenclature Revision for Encapsulated Follicular Variant of Papillary Thyroid Carcinoma: A Paradigm Shift to Reduce Overtreatment of Indolent Tumors. JAMA Oncol 2016;2:1023-9.

8. Chereau N, Greilsamer T, Mirallié E, et al. NIFT-P: Are they indolent tumors? Results of a multi-institutional study. Surgery 2019;165:12-6.

9. Hirokawa M, Higuchi M, Suzuki A, et al. Noninvasive follicular thyroid neoplasm with papillary-like nuclear features: a singleinstitutional experience in Japan. Endocr J 2017;64:1149-55.
10. Wong KS, Strickland KC, Angell TE, et al. The Flip Side of NIFTP: an Increase in Rates of Unfavorable Histologic Parameters in the Remainder of Papillary Thyroid Carcinomas. Endocr Pathol 2017;28:171-6.

11. Bychkov A, Keelawat S, Agarwal S, et al. Impact of non-invasive follicular thyroid neoplasm with papillary-like nuclear features on the Bethesda system for reporting thyroid cytopathology: a multi-institutional study in five Asian countries. Pathology 2018; 50:411-7.

12. Rana C, Vuong HG, Nguyen TQ, et al. The incidence of noninvasive follicular neoplasm with papillary like nuclear features: A meta-analysis assessing worldwide impact of the reclassification. Thyroid 2021;31:1502-13.

13. Lloyd RV, Osamura RY, Klöppel G, et al. (Eds). WHO Classification of Tumours of Endocrine Organs. 4th ed, Vol. 10. Lyon, France: World Health Organization; 2017.

14. Cibas ES, Baloch ZW, Fellegara G, et al. A prospective assessment defining the limitations of thyroid nodule pathologic evaluation. Ann Intern Med 2013;159:325-32

15. Gan TR, Nga ME, Lum JH, et al. Thyroid cytology-nuclear versus architectural atypia within the "Atypia of undetermined significance/follicular lesion of undetermined significance" Bethesda category have significantly different rates of malignancy. Cancer Cytopathol 2017;125:245-56.

16. Lim JXY, Nga ME, Chan DKH, et al. Subclassification of Bethesda Atypical and Follicular Neoplasm Categories According to Nuclear and Architectural Atypia Improves Discrimination of Thyroid Malignancy Risk. Thyroid 2018;28:511-21.

17. Steward DL, Carty SE, Sippel RS, et al. Performance of a Multigene Genomic Classifier in Thyroid Nodules With Indeterminate Cytology: A Prospective Blinded Multicenter Study. JAMA Oncol 2019;5:204-12.

18. Liu Y, Pan B, Xu L, et al. The Diagnostic Performance of Afirma Gene Expression Classifier for the Indeterminate Thyroid Nodules: A Meta-Analysis. Biomed Res Int 2019;2019:7150527.

19. Haugen BR, Alexander EK, Bible KC, et al. 2015 American Thyroid Association Management Guidelines for Adult Patients with Thyroid Nodules and Differentiated Thyroid Cancer: The American Thyroid Association Guidelines Task Force on Thyroid Nodules and Differentiated Thyroid Cancer. Thyroid 2016;26:1-133.

20. Ruanpeng D, Cheungpasitporn W, Thongprayoon C, et al. Systematic Review and Meta-analysis of the Impact of Noninvasive Follicular Thyroid Neoplasm with Papillary-Like Nuclear Features (NIFTP) on Cytological Diagnosis and Thyroid Cancer Prevalence. Endocr Pathol 2019;30:189-200.

21. Bychkov A, Hirokawa M, Jung CK, et al. Low Rate of Noninvasive Follicular Thyroid Neoplasm with Papillary-Like Nuclear Features in Asian Practice. Thyroid 2017;27:983-4.

22. Fonseca D, Bhuyan S, Murthy SS, et al. Noninvasive follicular thyroid neoplasm with papillary-like nuclear features: A distinct clinicopathologic entity. Indian J Pathol Microbiol 2018;61:380-2.

23. Sung H, Ferlay J, Siegel RL, et al. Global Cancer Statistics 2020: GLOBOCAN Estimates of Incidence and Mortality Worldwide for 36 Cancers in 185 Countries. CA Cancer J Clin 2021;71:209-49.

24. Nikitski AV, Rogounovitch TI, Bychkov A, et al. Genotype Analyses in the Japanese and Belarusian Populations Reveal Independent Effects of rs965513 and rs1867277 but Do Not Support the Role of FOXE1 Polyalanine Tract Length in Conferring Risk for Papillary Thyroid Carcinoma. Thyroid 2017;27:224-35.

25. Parente DN, Kluijfhout WP, Bongers PJ, et al. Clinical Safety of Renaming Encapsulated Follicular Variant of Papillary Thyroid Carcinoma: Is NIFTP Truly Benign? World J Surg 2018;42:321-6. 